\title{
NUMERICAL TEST OF MOLECULAR-FIELD RENORMALIZATION GROUP
}

\author{
G. KAMIENIARZ \\ Computational Physics Division, Institute of Physics, A. Mickiewicz University \\ Umultowska 85, 61-614 Poznani, Poland
}

A new transfer matrix approach has been worked out to test the predictions of the molecular-field renormalization group for square Ising clusters with a linear size up to $L \leq 11$. The convergence of the finite-size critical couplings towards the exact value for the molecular-field renormalization group is shown and the limit of the ratio $y_{h} / y_{t}$ consistent with the corresponding universal value is revealed.

PACS numbers: $64.60 . \mathrm{Ak}, 75.10 . \mathrm{Im}, 75.40 . \mathrm{Mg}$

The molecular field approximation has become a valuable tool for a qualitative understanding of phase diagrams of three-dimensional systems and for obtaining quantitative estimates of nonsingular thermodynamic properties. Its most serious drawback is the prediction of classical critical exponents. A successful attempt to improve this deficiency [1-3] is the idea of the molecular-field renormalization group (MFRG) which can be illustrated for the nearest-neighbour Ising model on the square lattice.

Consider two square clusters consisting of $N=L^{2}$ and $N^{\prime}=L^{2}$ sites, where $L$ and $L^{\prime}$ denote the linear size of a given cluster, with $L>L^{\prime}$. In the spirit of the mean-field theory, the border spins of the clusters are subject to the symmetry-breaking field $b$ and $b^{\prime}$, respectively. For the cluster of the size $L$, the Hamiltonian in the presence of a magnetic field can be written as

$$
-\beta H \equiv \mathcal{H}_{L}=K \sum_{\langle i, j\rangle} S_{i} S_{j}+h \sum_{j} S_{j}+K b \sum_{j} n_{j} S_{j},
$$

where $K$ si inds for the coupling $\beta J$ and the parameters $h$ and $b$ denote the external and the effective field, respectively. The first sum runs over the nearest-neighbour pairs, the second over all the sites and the third over the edge spins only, so that the factor $n_{j}$ counts the number of effective fields $\left(n_{j}=1\right.$ except for the corner spins where $n_{j}=2$ ).

In MFRG, the scaling behaviour of the cluster magnetizations $m$ and $m^{\prime}$ under rescaling $l=\left(N / N^{\prime}\right)^{1 / d}$ is imposed [1] 


$$
m^{\prime}\left(K^{\prime}, h^{\prime}, b^{\prime}\right)=l^{d-y_{h}} m(K, h, b)
$$

together with the ansatz $b^{\prime}=l^{d-y_{h}} b$.

A mapping $(K, h, b) \rightarrow\left(K^{\prime}, h^{\prime}, b^{\prime}\right)$ can be found from (1), setting $h=h^{\prime}=0$ and expanding both sites to the leading order in $b$ and $b^{\prime}$. Then the fixed point equation of the form

$$
\Theta^{\prime}\left(K^{*}\right)=\Theta\left(K^{*}\right)
$$

follows, where

$$
\Theta(K)=\left\langle\frac{1}{N} \sum_{i} S_{i} \sum_{j} n_{j} S_{j}\right\rangle
$$

denotes a two-point correlation function in the limit $h=b=0$. Once the fixed point $K^{*}$ is calculated, the thermal and the magnetic exponents $y_{t}$ and $y_{h}$ can be determined from the corresponding scaling fields.

This method of computation of non-classical critical properties of lattice models is attractive due to its efficiency and simplicity and can be easily applied $[4,5]$ to bulk critical phenomena as well as to surface, random and dynamic effects. Initially [1] the estimates of the non-classical critical exponents appeared to be inferior to the estimates of critical couplings. In a number of publications some attempts to improve this shortcoming have been undertaken by a redefinition of the rescaling factor [4], a two-step renormalization approach based on three clusters [2] or an idea of effective fields with correlations [6]. However, the validity of the basic assumption (1) has not been confirmed so far as the convergence of the MFRG results has not been systematically checked by using sequences of large cluster sizes. The main difficulty arises from summation over all the $2^{L \times L}$ Ising configurations in (3), blowing up quickly with increasing $L$. With some computational effort the renormalization-group calculations have been performed up to $L=6$ [4]. Considering the wide range of the MFRG applications, the test of convergence in the finite-size scaling region $1 / L \ll 1$ seems rather important.

In this paper we report a new transfer matrix approach which enables both the numerically exact solutions of Eq. (2) up to $L=11$ and the asymptotic analysis of the corresponding finite-size data.

The idea is as follows. Our system consists of a square cluster of size $L$ and contains $L \times L$ spins $S= \pm 1$. The spins on the external edges are subject to an effective field $b$. For the $L \times L$ cluster and small $h$ and $b$, the partition function $\mathcal{Z}_{L}$ can be expanded in a power series. The thermal expectation values $\Theta(K)$ present in Eq. (2) can be then evaluated from the ratios of the corresponding coefficients in the expansion of $\mathcal{Z}_{L}$. It appears that

$$
\Theta(K)=\mathcal{Z}_{1,1}^{(2)} / \mathcal{Z}_{0,0}^{(0)},
$$

where

$$
\mathcal{Z}_{m, n}^{(m+n)}=\left(\frac{\partial^{m+n} \mathcal{Z}_{L}}{\partial h^{m} \partial b^{n}}\right)_{h, b=0} .
$$

The computations of the partition function $\mathcal{Z}_{L}$ and its expansion are carried out by the powerful transfer matrix technique. If the spins belonging to the $j$-th 
column of the cluster are denoted as $S_{j}=\left(S_{j 1}, S_{j 2}, \ldots, S_{j L}\right)$, the partition function is given by

$$
\mathcal{Z}_{L}=\sum_{S_{1}, S_{2}} T_{L}\left(S_{1}, S_{2}\right)
$$

where the global transfer matrix $T_{L}$ is defined in the $2^{L}$-dimensional manifold of all the configurations of $L$ spins $S_{j k}(k=1, \ldots, L)$. The matrix $\boldsymbol{T}_{L}$ can be factorized into the product

$$
T_{L}=T_{0} T^{L-1} T_{0}
$$

where $T_{0}$ is the diagonal matrix defined by

$$
T_{0}\left(S_{1}, S_{2}\right)=\exp \left(K l \sum_{k=1}^{L} S_{1 k}\right) \delta_{S_{1}, S_{2}}
$$

and $T$ is a non-diagonal matrix which can be split into the product

$$
T=T_{v} T_{h} T_{v}
$$

with the diagonal matrix $T_{v}$ expressed by

$$
T_{v}\left(S_{i}, S_{j}\right)=\exp \frac{1}{2}\left[K \sum_{k=1}^{L-1} S_{j k} S_{j, k+1}+h \sum_{k=1}^{L} S_{j k}+K b\left(S_{j 1}+S_{j, L}\right)\right] \delta_{S_{i}, S_{j}} .
$$

The corresponding matrix $T_{h}$ is non-diagonal

$$
T_{h}\left(S_{i}, S_{j}\right)=\exp \left(K \sum_{k=1}^{L-1} S_{i k} S_{j k}\right)
$$

\section{TABLE}

Estimates of the critical couplings $K_{L L^{\prime}}^{*}$ and the critical exponents $y_{t}$ and $y_{h}$ for the square clusters with linear size $L$ and $L^{\prime}$.

\begin{tabular}{c|c|c|c|c|c}
\hline \hline$L$ & $L^{\prime}$ & $K_{L L^{\prime}}^{*}$ & $y_{t}$ & $y_{h}$ & $y_{h} / y_{t}$ \\
\hline 1 & & & & & \\
2 & 1 & 0.3609089 & 0.69287882 & 1.50000000 & 2.164880722 \\
3 & 2 & 0.3810807 & 0.77813108 & 1.56605949 & 2.012590848 \\
4 & 3 & 0.3930155 & 0.81631814 & 1.59784863 & 1.957384691 \\
5 & 4 & 0.4008624 & 0.83823988 & 1.61691985 & 1.928946461 \\
6 & 5 & 0.4064323 & 0.85248095 & 1.62981442 & 1.911848494 \\
7 & 6 & 0.4106017 & 0.86247487 & 1.63920749 & 1.900585799 \\
8 & 7 & 0.4138456 & 0.86986741 & 1.64640276 & 1.892705418 \\
9 & 8 & 0.4164448 & 0.87555168 & 1.65211770 & 1.886944812 \\
10 & 9 & 0.4185761 & 0.88005235 & 1.65678164 & 1.882594416 \\
11 & 10 & 0.4203567 & 0.88370006 & 1.66066940 & 1.879222912 \\
\hline$\infty$ & & $0.441(1)$ & $0.912(1)$ & $1.66(1)$ & $1.866(1)$
\end{tabular}


but can be expressed as a product of sparse matrices convenient for numerical computations.

Using our approach, we have solved the fixed point Eq. (2) for $K^{*}$ with the accuracy up to 7 decimal places for $2 \leq L \leq 11$ and $L^{\prime}=L-1$. The final results for $K^{*}$ as well as for the critical exponents $y_{t}$ and $y_{h}$ are given in the corresponding columns of Table. The values for $L \leq 6$ coincide with those previously published to all the decimal placed quoted.

The series given in Table have been analyzed by a number of extrapolation techniques to calculate the limit for $L \rightarrow \infty$. The best convergence has been found for the alternating $\varepsilon$-algorithm and these estimates are given as the extrapolated values in the last row of Table. The uncertainties on the last decimal places are written in parentheses.

According to the data in Table, the limit $K_{\mathrm{c}}$ of the series for the critical couplings $K_{L L^{\prime}}^{*}$ coincides with the exact Ising value 0.4406868 within the quoted error bar. The corresponding series for $y_{t}$ and $y_{h}$ do not seem to converge towards the exact values. Within MFRG the ratio $y_{h} / y_{t}$ deserves special attention. This quantity is independent of the definition of the rescaling factor [4] and is expected to show the best convergence for $L \rightarrow \infty$. We confirm this behaviour, although the limit deviates slightly from 1.875 .

I would like to thank G. Musiat and P. Pawlicki for valuable discussions as well as the Supercomputing and Networking Center in Poznan for an access to Cray YMP-EL and Cray J916. The work has been supported in part by the Committee for Scientific Research via grant 2 P302 11606.

\section{References}

[1] J.O. Indekeu, A. Maritan, A. Stella, J. Phys. A 15, L291 (1982).

[2] J.O. Indekeu, A. Maritan, A. Stella, Phys. Rev. B 35, 305 (1987).

[3] I.P. Fittipaldi, J. Magn. Magn. Mater. 131, 43 (1994).

[4] K. Croes, J.O. Indekeu, Mod. Phys. Lett. B 7, 699 (1993).

[5] J.R. Sousa, D.F. de Albuquerque, Phys. Rev. B 48, 16484 (1993).

[6] G. Kamieniarz, R. Dekeyser, in: Symmetry and Structural Properties of Condensed Matter, Eds. W. Florek, D. Lipiński, T. Lulek, World Scientific, Singapore 1993, p. 181. 